\title{
Manufactura de una estructura de órtesis dinámica para la dorsiflexión de la mano mediante modelado por deposición fundida
}

Juan Carlos Vásquez Jiménez

jcvazquezj@hotmail.com

Felipe Ernesto Orozco Contreras

ing.felipeorozco@hotmail.com

Asunción Adriana Arango Perdomo

asuncionadriana.ap@tehuacan.tecnm.mx

Serafín Reyes García

serafin.rg@tehuacan.tecnm.mx

María de Jesús Oregán Silva mariadejesus.os@tehuacan.tecnm.mx

Tecnológico Nacional de México / Instituto Tecnológico de Tehuacán División de Estudios de Posgrado e Investigación

Departamento de Metal - Mecánica

Tehuacán, Puebla, México

\section{RESUMEN}

El propósito de este trabajo es realizar la estructura base del prototipo de órtesis para la rehabilitación de los dedos de la mano, en el cual se recrean los movimientos de los cincos dedos por mediante mecanismos a través de servomotores que activaran independientemente los dedos de la mano tratando lograr una movilidad adecuada de la mano con problemas. Para el proceso de diseño se utiliza un software de diseño asistido por computadora $\mathrm{CAD}$, con el que se obtienen las piezas de manera virtual, se lleva a cabo un análisis de esfuerzos en las piezas que tienen sometimiento y para la manufactura de las partes necesarias para la órtesis es mediante el Modelado de Deposición Fundida MDF utilizando un material plástico de origen vegetal PLA y liviano. La órtesis debe de ser capaz de emular el movimiento de los dedos de la mano. Se manejará el movimiento de estos servomotores con un controlador, donde se memorizarán ejercicios recomendados para la rehabilitación de personas con daños motrices en la mano. Se 
consideran las medidas de la mano contempladas dentro con la Norma DIN 33 402, con la finalidad de diseñar un exoesqueleto tipo guante el cual estará constituido por varios mecanismos de plancha para el movimiento de cada uno de los dedos independiente. Se pretende con este proyecto minimizar al máximo los costos, logrando una órtesis con economía de bajo presupuesto para accesibilidad al público, obteniendo los materiales de fácil acceso y bajo costo.

Palabras clave: estructura; órtesis dinámica; modelado por deposición fundida. 


\title{
Design and manufacture using MDF of a dynamic orthosis structure for the fingers of the hand
}

\begin{abstract}
The purpose of this work is to make the base structure of the prototype of orthosis for the rehabilitation of the fingers of the hand, in which the movements of the five fingers are recreated by means of mechanisms through servomotors that independently activate the fingers of the hand. Trying to achieve adequate mobility of the hand with problems. For design process is used, CAD Computer-Aided Design software, with which the parts are obtained in a virtual way, a stress analysis is carried out in the parts that have subjection and for the manufacture of the necessary parts to the orthosis is by means of the Fused Deposition Modeling FDM using a plastic material light of vegetal origin PLA. The orthosis must be able to emulate the movement of the fingers of the hand. The movement of these servomotors will be operated with a controller, where recommended exercises for the rehabilitation of people with motor injuries in the hand will be memorized. The measurements of the hand contemplated within the DIN 33402 standard are considered, in order to design a glove-type exoskeleton which will be made up of several plate mechanisms for the movement of each of the fingers independently. It is intended with this project to minimize costs as much as possible, achieving an orthosis with a low budget economy for accessibility to the public, obtaining materials that are easily accessible and low cost.
\end{abstract}

Keywords: structure; dynamic orthosis; fused deposition modeling

Artículo recibido: 02 noviembre. 2021 Aceptado para publicación: 28 noviembre 2021 Correspondencia: jcvazquezj@hotmail.com Conflictos de Interés: Ninguna que declarar 


\section{INTRODUCCIÓN}

La órtesis es un mecanismo ortopédico o exoesqueleto, que se implementa en una parte del cuerpo de la persona, para contrarrestar, prevenir o corregir alguna anormalidad, para posibilitar una función (Levy, 2003). Zambudio (2009) explica que la palabra órtesis es utilizada para definir todos aquellos aparatos externos que sirven como ayuda, soporte y se utilizan en el campo de la ortopedia. El significado proviene del término griego orthos, que quiere decir recto que se puede entender como enderezar.

Las órtesis se pueden clasificar según su función en cuatro tipos: estabilizadoras o inmovilizadoras, que mantienen una posición e impiden movimientos indeseados, si el objetivo es actuar como soporte de un segmento paralizado, o para disminuir la amplitud articular de un segmento inflamado y doloroso. El grado de inmovilización deseado varía según el tipo de órtesis utilizado. Son las que tienen mayor utilidad; funcionales: llamadas también dinámicas, ya que llevan incorporado un elemento elástico que permite movilizar un segmento de un miembro paralizado; correctoras: sirven para corregir una deformidad esquelética, son más efectivos si se utilizan durante el desarrollo infantil y protectoras: mantienen la alineación de un miembro enfermo o lesionado. En cuanto a la extremidad, articulación o región anatómica a la que van a aplicar se clasifican en: extremidad superior: brazo, codo, mano; extremidad inferior: pie-tobillo-pantorrilla; rodilla; muslocadera (férulas o arneses); funcional o adaptativa del pie (plantilla y calzado ortopédico) y dorsolumbar. (“Órtesis y férulas”, s. f.)

El proyecto completo de la órtesis, está constituido de dos etapas, en la primera parte se diseña y se manufactura la estructura o armazón mediante la deposición fundida de material plástico y muy ligero y en la segunda parte se desarrolla su sistema de control el cual se basa en la implementación de un controlador Arduino, cuya retroalimentación se obtendrá por medio de lenguaje de programación, creando una secuencias de ejercicios programadas en el mecanismo de unos servomotores que moverán los dedos de la mano, para la simulación de dicha mano, el mecanismo automatizado se determinara el grado de amplitud para articular los falanges de la mano, creando un programa que pueda interactuar con el paciente y el especialista a través de una aplicación de celular. Para efectos de este trabajo solamente nos ocupamos de la manufactura de la armazón base de la órtesis. 
En este trabajo se establece el proceso de diseño para lo que se utiliza un software de diseño asistido por computadora $\mathrm{CAD}$, con el que se obtienen las piezas de manera virtual, se lleva a cabo un análisis de esfuerzos en las piezas mediante la aplicación CAE que tienen sometimiento de fuerzas y de la manufactura de las partes necesarias para la órtesis es mediante el Modelado de Deposición Fundida MDF utilizando un material plástico de origen vegetal PLA y liviano. Se realiza el ensamble virtual y se llevan a cabo las pruebas y ajustes antes de pasar al proceso de modelado.

El enfoque del prototipo es ayudar y colaborar con la incapacidad motriz de los dedos de la mano. Para el tratamiento de lesiones y discapacidades, se tratan mediante terapias físicas, ocupacionales o uso de aparatos ortopédicos y en algunos casos con cirugía. De los anteriores tratamientos el que mejor se adapta a las necesidades del paciente con problemas de movimiento muscular en las manos, se recomienda las órtesis, que brindan el apoyo necesario. Se pretende atacar el problema con el prediseño de una órtesis de rehabilitación, la cual está orientada a ser una herramienta como práctica para los fisioterapeutas, especialistas y principalmente para pacientes con la discapacidad, logrando avances en su rehabilitación.

El prototipo de la (órtesis) se ha pensado en diseñarlo en la comodidad y economía de las personas a las que éste va dirigido. Por lo tanto, sobre salen algunos beneficios que se mencionan a continuación.

- Fisiológicos: El prototipo de órtesis dará rehabilitación a los dedos de la mano que tengan dificultad de movilidad.

- Accesibilidad: Por su amigable uso, diseño y costo, hacer este prototipo es ideal para su uso profesional en el área de la salud de cualquier hospital o centro de rehabilitación CRI (Centro de Rehabilitación Integral), y también para cualquier usuario independiente que lo use desde la comodidad de su hogar.

- Económicos: El prototipo de órtesis automatizada, está diseñado para que la adquisición de esta órtesis esté al alcance de todas las personas, ya que todo está producido con materiales de fácil obtención reduciendo al más mínimo costo.

El objetivo de este proyecto es el de diseñar por un software CAD la estructura o armazón de una órtesis dinámica para la rehabilitación de los dedos de la mano y manufacturarla mediante el Modelado por Deposición Fundida MDF utilizando ácido láctico o poliácido láctico PLA. 


\section{ESTRATEGIAS METODOLÓGICAS, MATERIALES Y MÉTODOS}

\subsection{Antecedentes}

La órtesis dinámica de mano para la flexión de muñeca con doblado reverso de nudillos para la rehabilitación de personas con problemas motrices es diseñada con el fin de fortalecer gradualmente la flexión de dedos, posteriores a cirugía de flexores, evita la deformidad en flexión de dedos. Por lo regular en la muñeca se monta una abrazadera dinámica de aleación de aluminio, resorte de torsión, almohadilla de tela de esponja y materiales de lámina de goma de arco transpirable, uso más cómodo, más conveniente. Depende del resorte de tensión en el soporte de aleación de aluminio, los dedos y las articulaciones de la muñeca ahorran dorsiflexión puede tener actividades al mismo tiempo (Arce, 2005).

Se utiliza en el proceso de recuperación o rehabilitación en lesiones como parálisis del nervio radial, ruptura de ligamentos interóseos de los dedos, cicatriz palmar por quemaduras, considerando también la protección de la extremidad lesionada, mediante la dorsiflexión de la muñeca, con doblado reverso de nudillos para mantener en extensión las articulaciones metacarpofalángicas y balancín para extender las articulaciones interfalángicas distales, considerando una rehabilitación pertinente para que se tenga una recuperación favorable. (Ortiz et al, 2016)

\section{Los productos que se encuentran en el mercado son los siguientes:}

La empresa Ortopedia Nodal presenta una combinación de férula de resorte Oppenheimer para la dorsiflexión d la muñeca con doblado reverso de nudillos. Extiende las articulaciones metacarpofalángicas y balancín para extender las articulaciones interfalángicas distales. Indicada para parálisis radial. Además de una férula con barra lumbrical alta y baja, con cabestrillos y elásticos para cada uno de los dedos, de acuerdo con la prescripción médica. Elaborada sobre medidas en material termoplástico, lámina de aluminio y platina con ajuste en velcro (“Férula dinámica para mano”, s.f.). 
Figura 1. Férula dinámica con resorte Oppenheimer para extensión dedos.

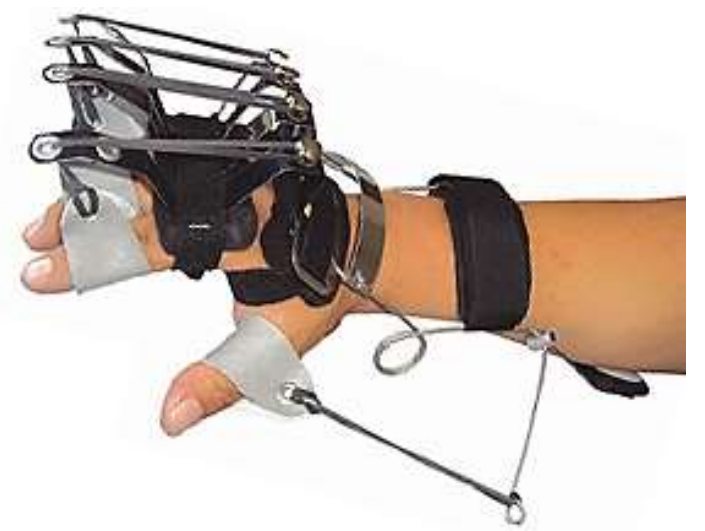

Fuente: http://ortopedianodal.com/producto/ferula-dinamica-para-flexion-de-munecay-dedos-mod-13b/

- La empresa Central Orthomedica presenta una férula de resorte de Oppenheimer para dorsiflexionar la muñeca con doblado reverso de nudillos, para extender las articulaciones metacarpofalángicas y balancín para extender las articulaciones interfalángicas distales. Indicada en la rehabilitación de una parálisis radial ("Férula dinámica", s.f.).

Figura 2. Férula de resorte de Oppenheimer para dorsiflexionar la muñeca con doblado reverso de nudillos

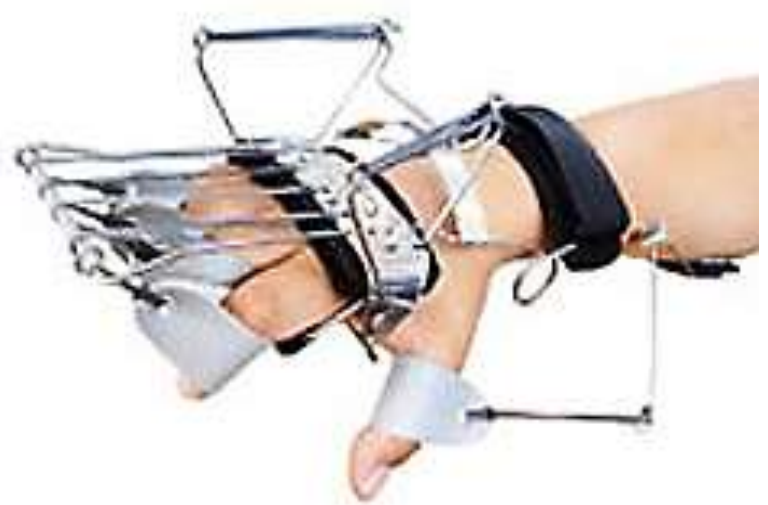

Fuente: https://www.centralorthomedica.com/brazo.html

\subsection{Propuesta del prototipo}

Algunas consideraciones recomendadas en el Manual de Órtesis que se deben de tener en cuenta para el diseño; las necesidades y características del usuario, el tipo de lesión y gravedad de ésta, y una vez realizada la órtesis se requiere mantener un proceso evaluativo colaborativo continuo. (Hospital del Trabajador, 2013) 
- Edad

- Nivel de actividad (facilita o dificulta la rutina ocupacional).

- Situación social y apoyo familiar

- Tiempo de uso y tolerancia al esfuerzo

- Tiempo y costo de fabricación

- Fácil de usar y retirar

- Motivación y capacidad de seguimiento de instrucciones

- Medidas corporales

- Proximidad al centro de atención y control

- Programa de ejercicios posible y tolerancia al dolor

- Simpleza de diseño

- Estética

Este proyecto está basado en un prototipo de diseño de exoesqueleto, que se la harán diferentes pruebas para ver qué tan eficaz es su mecanismo y su funcionamiento por medio del sistema de polea excéntrica biela-palanca ("Mecanismos", 2012).

El dispositivo desarrollado busca mantener los rangos articulares de la mano en su magnitud apropiada, así como mantener músculos y tendones en movimiento; de acuerdo con las necesidades de cada paciente, el barrido angular del movimiento debe cambiar para adaptarse a éste (Fique y Aponte, 2008)

Con el fin de mover las falanges de la mano para abrir y cerrar. Por medio de un servo motor clocado en la mano para realizar dicha función. Es útil para personas que tienen dificultad de movimiento de mano y sensibilidad a la punta de los de dos, pero no tienen suficiente fuerza para la movilidad de la mano en su conjunto

Figura 3. Propuesta de para la estructuración de la órtesis

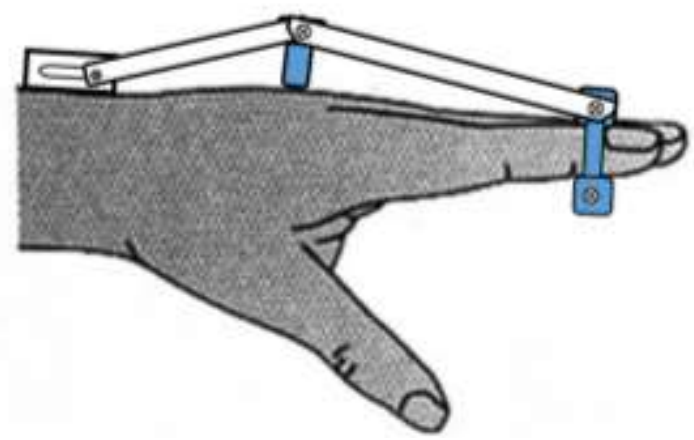

Imagen base tomada de: http://introduccionalapm.blogspot.com/2011/05/articulacionde-manos-y-dedos.html 
La tecnología desarrollada para el prototipo se caracteriza por ser un exoesqueleto útil para personas que tienen movimiento de mano y sensibilidad a la punta de los dedos pero no tienen suficiente fuerza para soportar los objetos.

La órtesis cabe destacar que: puede ser utilizada por cualquier persona que tenga movilidad y sensibilidad, pero no fuerza en la mano, puede adaptarse a diferentes tamaños de mano, su accionamiento va a depender de energía externa por medio de servomotores, facilita las actividades de la vida diaria, su peso es muy reducido, y estéticamente puede mimetizarse con un guante y su fabricación es fácil y económica, y su uso es muy fácil (Peña; Ticó; López, Abenoza y Romero, s.f.).

\subsection{Diseño de las piezas de la órtesis mediante un software CAD}

En este punto se considera el diseño mediante un software CAD de las diferentes tipos de piezas independientes que conformarán la órtesis en su complejidad mostrando cada una de ellas con las medidas específicas de una mano para después pasar a un ensamble de función de mecanismo (Gómez, 2010):

a. Anillo sujetador de dedo. El anillo de sujeción del dedo de la mano, este anillo tiene la función de entrar en los dedos de la mano, tiene una peque abertura en la parte inferior de el con unos barrenos a los extremos, esto se realizó con el fin de autoajustar el anillo a todos los dedos, colocando un tornillo y su tuerca para realizar la acción de apretar y de aflojar a como lo pide el dedo, en la parte de arriba se encuentra otro barreno el cual va conectado a una extensión de la parte del exoesqueleto con el fin de tener el movimiento al abrir o cerrar el dedo, a si logrando crear un tipo de palanca sobre el dedo y crear el movimiento. Este anillo se le hicieron diferentes tipos de chaflán sobre los bordes, con el fin de dejarlo lo más curveado posible, para un deslizamiento perfecto, para que la superficie de los dedos logre un mayor deslizamiento o facilidad de entrada y salida al colocárselo a dicho paciente.

b. Sujetador de placa e inmovilizador en muñeca. La pieza se encuentra en forma de U, que este va colocado en la parte de abajo del soporte de placa impresa y baterías el cual este va a atornillado junto con la placa, haciendo presión para inmovilizar todo el cuerpo en caso de movimientos.

c. Biela de movimiento. Esta pieza es fundamental para el movimiento del mecanismo de cada dedo, ya que esta va conectada al engrane del servomotor junto con una arandela 
de plástico, lo que hace que se mueva el mecanismo de apertura y cerradura del exoesqueleto de cada dedo independiente.

d. Extensor largo para anular y dedo medio. En esta parte podremos observar el extensor largo solo lo ocupan los dedos de en medio, que son el anular y dedo medio, ya que los servomotores de este mecanismo se encuentras por delante de los demás, lo cual es más corto el mecanismo, no como los dedos índice y meñique el cual su mecanismo es más extenso por la posición de servomotores, ocupan dos. La forma de los barrenados de en medio del extensor, hacen que sea más ligero, al mismo tiempo ahorren material de impresión el cual el mismo cuerpo resiste las diferentes fuerzas ejercidas sobre los dedos, ya que esto le permite que el punto de ruptura sea menor al de una pieza completa sólida.

e. Extensor corto para meñique, índice y pulgar. El extensor pequeño es apto para los dedos mencionados, ya que estos se encuentran conectados a otro extensor corto, por la posición de los servomotores que se encuentran en la parte trasera del soporte posición excepto el del dedo pulgar, este solo requiere uno de estos extensores ya que el dedo es corto así como la posición del servo es más cercana a la del pulgar.

f. Extensión de mecanismo para índice y meñique. Esta pieza es una extensión de solo dos dedos, que son el dedo meñique y el dedo índice, el cual se tuvo que hacer por la posición de los servomotores, los servomotores tienen la limitación de movimiento, la limitación se debe al espacio del soporte que se redujo en los más mínimo, ya que no todos tienen la misma posición, ya que esto permite que el mecanismo se extienda entre los demás, tenga funcionalidad de movimiento del exoesqueleto de los dedos mencionados.

Figura 4. Piezas de la estructura de la órtesis.

\begin{tabular}{|c|c|c|}
\hline $\begin{array}{l}\text { a. Anillo sujetador de } \\
\text { dedo }\end{array}$ & $\begin{array}{l}\text { b. Sujetador de placa e } \\
\text { inmovilizador en } \\
\text { muñeca }\end{array}$ & c. Biela de movimiento \\
\hline
\end{tabular}




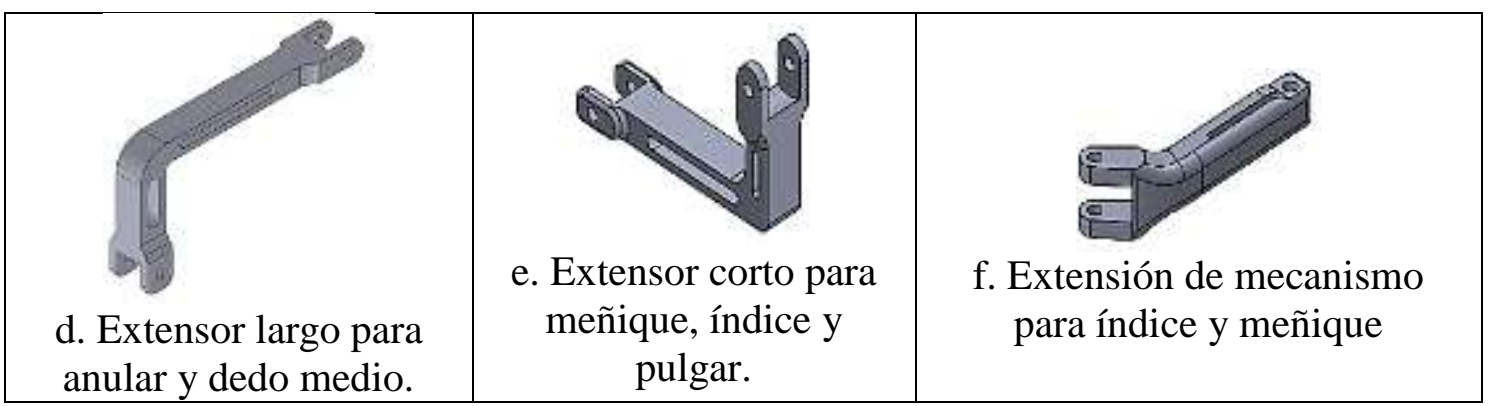

g. Soporte del cuerpo de la órtesis. La siguiente figura que se muestra es el cuerpo de todo el exoesqueleto, donde se observa que tiene tres rieles, en esta parte superior, es donde se insertaran tornillos de 2 1/2" pulgas de largo por 1/8" en cuerda estándar, que estarán colocados de manera vertical donde la cabeza del tornillo entrara por el riel quedando de forma que la punta quede arriba, para que en esa forma entren los sujetadores de los servomotores motores. La parte lateral izquierda se encuentran unos barrenos con bisagras, en esta parte se colocará otra base la cual sujetará el otro servomotor que será la parte del dedo pulgar. El cual tendrá la capacidad de moverse hacia arriba o hacia abajo, como lo pida el dedo pulgar del paciente.

h. Soporte del servo del dedo pulgar. Se puede observar que la siguiente pieza dibujada, que se tiene solamente dos rieles para la sujeción de un solo servomotor, el cual corresponde al del dedo pulgar, así como también se pueden observar las bisagras con sus barrenos, ya que estas entraran sujetas al otro soporte y se insertara una varilla de $1.2 \mathrm{~mm}$ entre los dos soportes para su agarre, teniendo la movilidad para ajustarse al dedo pulgar.

i. Sujetador de servomotores. El sujetador de los servomotores esta echo en forma de canal $\mathrm{C}$ el cual adquiere la forma de los servos motores, su función es mantenerlos en su posición, que respectivamente están sujetos en la base, ya que esta cuenta con los barrenos laterales, el cual van insertados junto con los tonillos que se colocaron en el riel del soporte, colocándolos con rondanas de presión y sus tuercas para su agarre.

j. Tapa de porta pilas. La siguiente figura se muestra la tapa de la porta pilas, el cual las protege de algún daño externo, así como la caída de las baterías ya que en la mano se encuentra en constante movimiento con el uso de la órtesis.

k. Base de porta pilas e impresión de placa. En la siguiente figura se muestra el soporte que llevara la placa de impresión (PCB) junto con los componentes, que son el Arduino la antena Bluetooth, así como las baterías doble A de 4.7 volts a 5800 miliAmperes. 
Figura 5. Piezas de la estructura del sistema motriz y control.

\begin{tabular}{|c|c|c|}
\hline $\begin{array}{c}\text { g. Soporte del cuerpo de la } \\
\text { órtesis }\end{array}$ & $\begin{array}{l}\text { h. Soporte del servo del dedo } \\
\text { pulgar }\end{array}$ & $\begin{array}{l}\text { i. Sujetador de } \\
\text { servomotores }\end{array}$ \\
\hline j. Tapa de porta pilas & k. Base de porta pilas e impresión & \\
\hline
\end{tabular}

\subsection{Ensamble virtual de las piezas de la órtesis en software CAD}

En esta parte se puede observar las capturas de pantalla, donde se muestran mostrar las diferentes posiciones y ángulos del exoesqueleto ensamblado, el cual el objetivo del montaje, es demostrar la complejidad del movimiento con la que se puede desplazar los diferentes mecanismo de cada dedo, así mismo viendo la construcción de todos sus componentes, la finalidad es que el primer prototipo de exoesqueleto, logre la movilidad de los dedos de la mano en un promedio del 50 al 70 por ciento de eficiencia, ya que las personas con discapacidad fatal inmovilidad solo logran un 10 a 30 porciento de movilidad de su mano independientemente.

Figura 6. Vistas de la órtesis ensamblada.

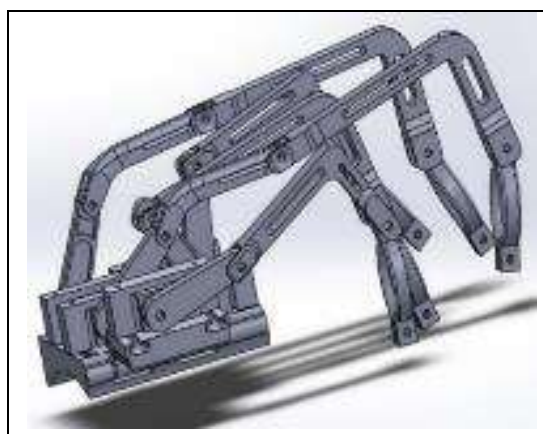

Soportes de los dedos extendidos

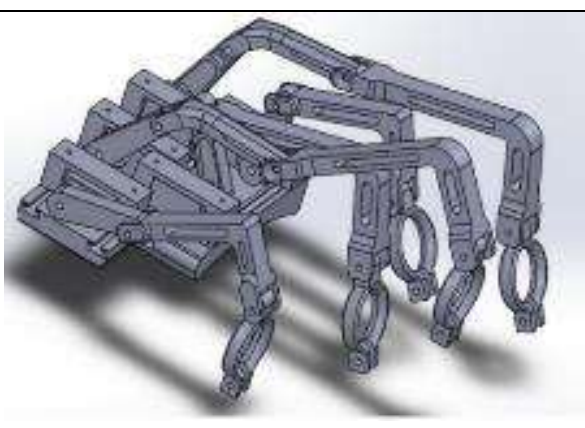

Soportes de los dedos retraídos 


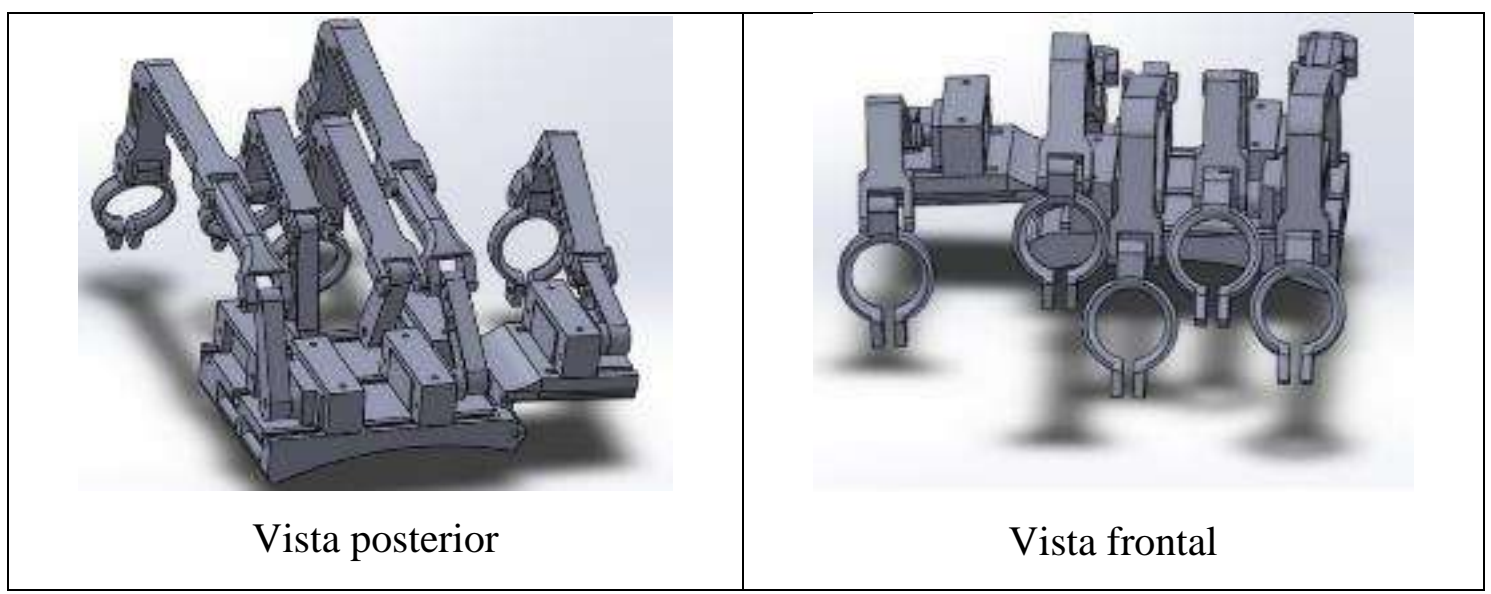

\section{APLICACIÓN DEL MODELADO POR DEPOSICIÓN FUNDIDA}

\subsection{Introducción}

La manufactura aditiva mediante el modelado por deposición fundida mejor conocido como impresión 3D es un proceso muy fácil, aplicado desde principiantes y utilizado hasta en sistemas de producción, en el cual se pueden ocupar una variedad de materiales y colores con tiempos de respuesta rápidos y una amplia gama de materiales y colores destinados a aplicaciones funcionales y verificación de diseño (Cervantes, 2019).

La patente inicial de FDM (Fused Deposition Modeling o también conocido como FFF de igual forma Fused Filament Fabrication que es Fabricación con Filamento Fundido) fue otorgada al fundador de la empresa Stratasys, Scott Crump en 1992. La resistencia de FDM está en la gama de materiales y las propiedades mecánicas efectivas de las piezas resultantes fabricadas con esta tecnología. Las piezas fabricadas se encuentran entre las más resistente para cualquier proceso de fabricación aditiva a base de polímeros (Gibson, Rosen and Stucker, 2015).

Es una técnica considerada como la más sencilla, la tecnología de modelado por deposición fundida MDF se basa en tres elementos principales: una placa/cama de impresión en la cual se imprime la pieza, una bobina de filamento que sirve como material de aportación para la impresión y un cabeza de extrusión también llamada extrusor. En resumen, el filamento es jalado por la impresora y fundido por el extrusor, que deposita el material de forma precisa capa por capa sobre la placa de impresión. Estas capas luego se enfrían y solidifican, lo que da como resultado una parte impresa que ofrece una alta durabilidad y resistencia al calor. La FDM requiere un material de soporte de sacrificio que se deposita a través de una segunda boquilla. La impresión 3D comienza cuando la 
máquina alcanza una temperatura necesaria para la fusión del material. Entre los materiales de impresión 3D más populares en la deposición por fusión se encuentran el PLA (Ácido Poliláctico) y el ABS (Acrilonitrilo Butadieno Estireno). (Sánchez, 2017)

\subsection{Material de impresión de la órtesis: filamento ácido poliláctico (PLA)}

Los filamentos PLA son recomendados por su facilidad para imprimir, compatibles con prácticamente cualquier impresora 3D y pluma 3D. El PLA contiene aditivos plastificantes, que, por ser más caros, suelen ser usados únicamente en filamentos de alta calidad. Estos aditivos prolongan la vida útil del producto y mejoran significativamente las impresiones 3D. Cada milímetro de filamento es probado por sistemas de control láser, garantizando un diámetro estable y un producto libre de burbujas.

Se enrolla en su carrete usando sistemas computarizados, que proporcionan una distribución ordenada, concediendo así una conservación adecuada del filamento, ya que evita el maltrato y deformaciones. Además, anula la posibilidad de rupturas por enredos durante las impresiones ("PLA-blanco", s.f.).

Tabla 1. Ficha técnica de PLA adquirido.

\begin{tabular}{|l|c|}
\hline \multicolumn{1}{|c|}{ Características } & Datos \\
\hline Diámetro & $1.75 \mathrm{~mm}$ \\
\hline Peso & $1 \mathrm{~kg}$ \\
\hline Color & Blanco \\
\hline Temperatura de impresión & $190-230^{\circ} \mathrm{C}$ \\
\hline Temperatura de la cama & $50-65^{\circ} \mathrm{C}$ \\
\hline Densidad & $1.24 \mathrm{~g} / \mathrm{cm}^{3}$ \\
\hline Tolerancia de diámetro & $\pm 0.03 \mathrm{~mm}$ \\
\hline
\end{tabular}

\subsection{Equipamiento utilizado: Impresora 3D ANET A8}

La impresora por utilizar es la ANET A8 Cuenta con una pantalla LCD 2004 con cinco botones para una impresión sencilla y cómoda. Eso sí, sacarle todo el partido requiere tiempo, especialmente por su calibración manual, pero una vez conseguido se obtiene una calidad alta de impresión (“Anet A8”, s.f.). 
Tabla 2. Características de la impresora 3D ANET A8.

\begin{tabular}{|l|l|}
\hline \multicolumn{1}{|c|}{ Características } & \multicolumn{1}{c|}{ Datos } \\
\hline Resolución & $\pm 0.1-0.2 \mathrm{~mm}$ \\
\hline Velocidad Movimiento & $10 \sim 100 \mathrm{~mm} / \mathrm{s}$ \\
\hline Temperatura. Extrusor & $250^{\circ} \mathrm{C}$ \\
\hline Temperatura Cama & $100^{\circ} \mathrm{C}$ \\
\hline Filamento Preferencial & $\mathrm{PLA}$ \\
\hline Volumen Impresión & $220 \times 220 \times 240 \mathrm{~mm}$ \\
\hline Formato Archivo & $\mathrm{STL}, \mathrm{OBJ}, \mathrm{JPG}$ \\
\hline Material Cama & Aleación de aluminio \\
\hline Diámetro Filamento & $1.75 \mathrm{~mm}$ \\
\hline Voltaje & $110-220 \mathrm{~V}$ \\
\hline Potencia & $240 \mathrm{~W}$ \\
\hline Tamaño Artículo & $50 \mathrm{X} 45 \mathrm{X} 40 \mathrm{~cm}$ \\
\hline Peso & $8 \mathrm{Kg}$ \\
\hline
\end{tabular}

Figura 7. Impresora $3 D$ ANET A8

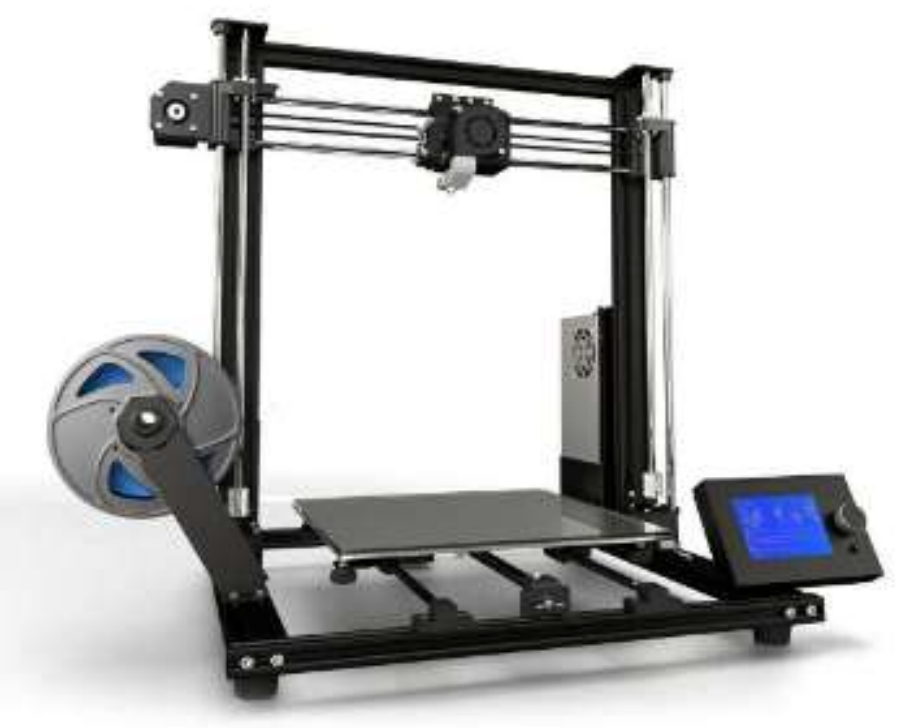

\subsection{Proceso de impresión 3D en tiempo real}

En esta parte se mostrarán imágenes de las impresiones realizadas de las piezas del exoesqueleto en tiempo real, solo como comprobación, resultado y demostración del funcionamiento de impresión, así como la impresora que se utilizó para dicho proyecto durante el proceso y programa. 
Solo se mostrarán algunas imágenes de las piezas impresas, el promedio mínimo de la pieza más pequeña fue de 2 horas, así como el tiempo máximo por la más grande fue de 9 horas haciendo total de más 180 horas de impresión continua por todo el proyecto. El ajuste de esta impresora tiene un margen de error de 0.2 milímetros entre las capas de impresión.

Figura 8. Se puede observar el cabezal imprimiendo capa por capa del soporte de los servos. En la pantalla LCD se muestra el tiempo, temperatura y las coordenadas de posicionamiento del cabezal.

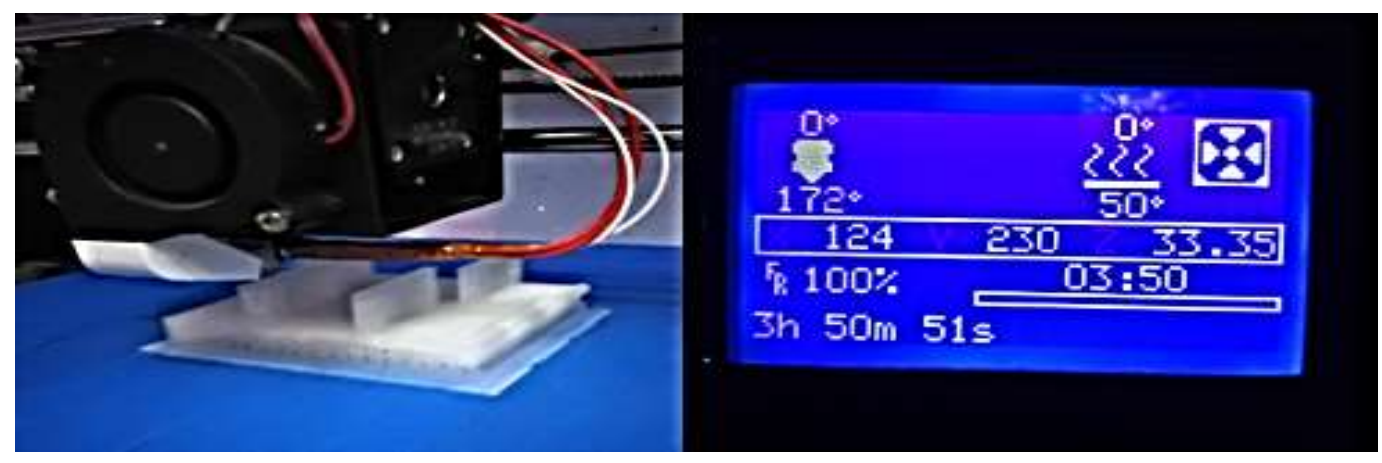

Figura 9. Se observa la impresión del anillo de sujetador de dedo, este anillo se hizo con una abertura en el centro para que fuera auto ajustable.

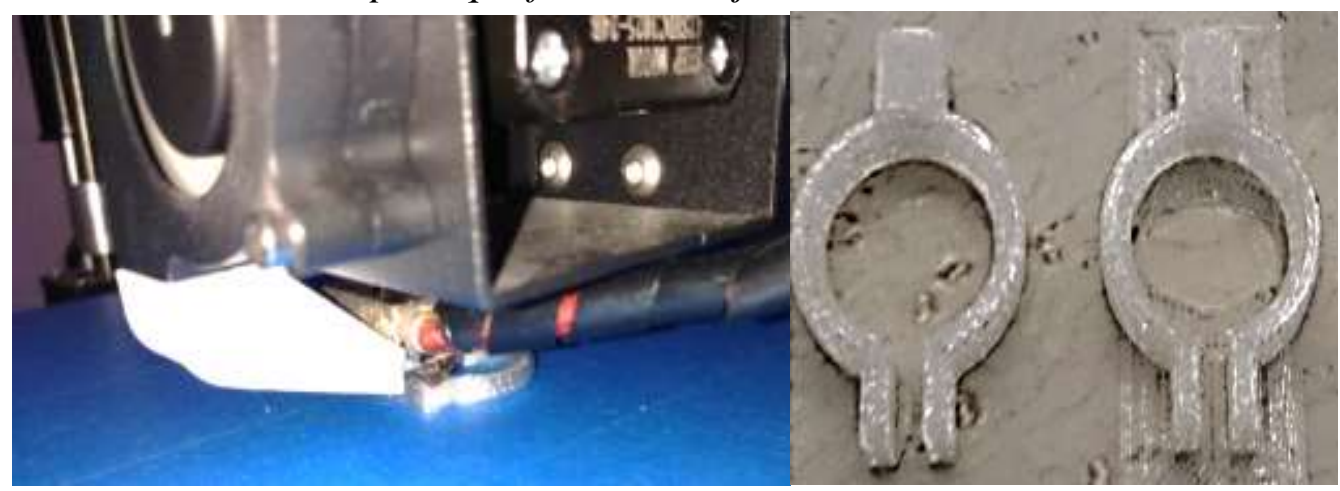

Figura 10. Soporte de servomotores con dos entradas para la entrada de una banda de sujeción y la base de soporte de servo para el dedo pulgar.

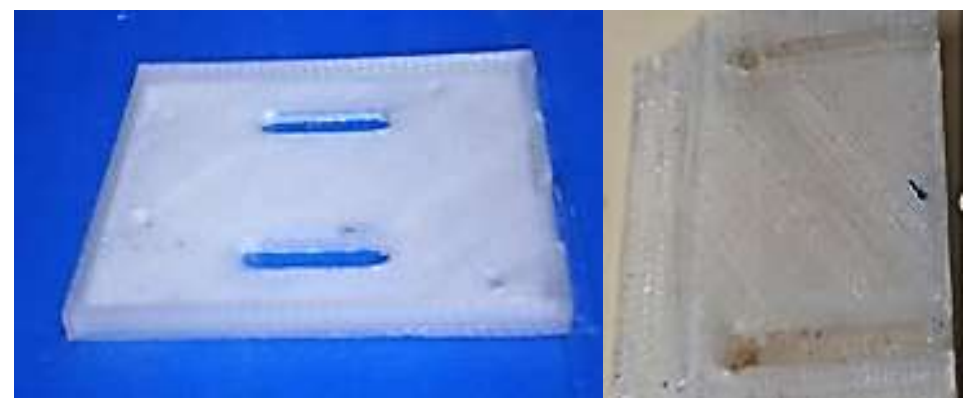


Figura 11. Piezas impresas, bielas de los servomotores, así como el soporte.

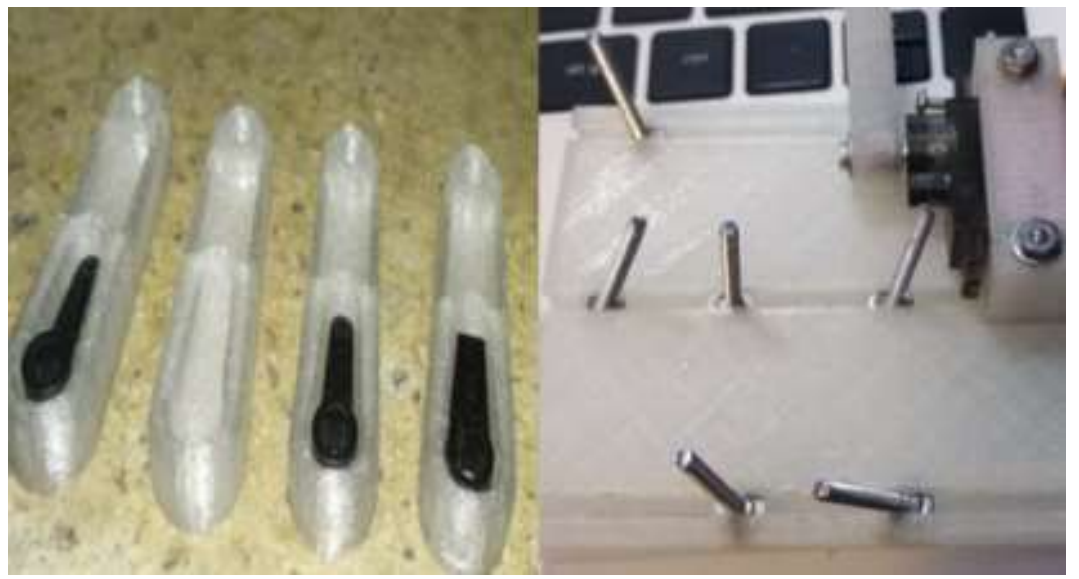

\subsection{Montaje del cuerpo del exoesqueleto.}

Figura 12. Se muestra extensor largo que va en los tres dedos del centro de la mano, así como los extensores pequeños que van en el pulgar y el meñique

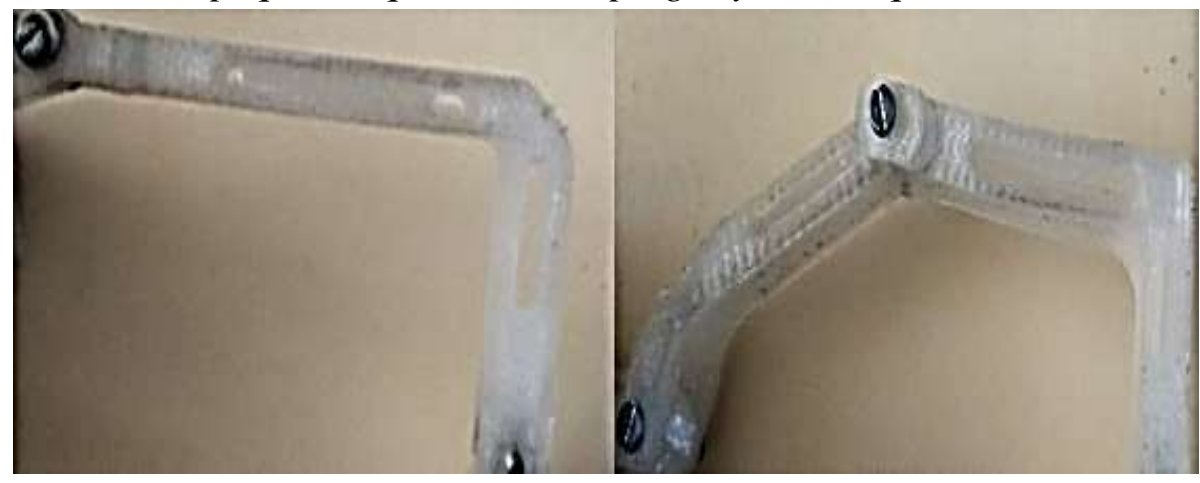

Figura 13. Se observa la órtesis ya conformada por todas las piezas impresas, el guante se le puso para en caso de que la mano fuera más delgada o pequeña u este entre de manera justa sobre la órtesis.

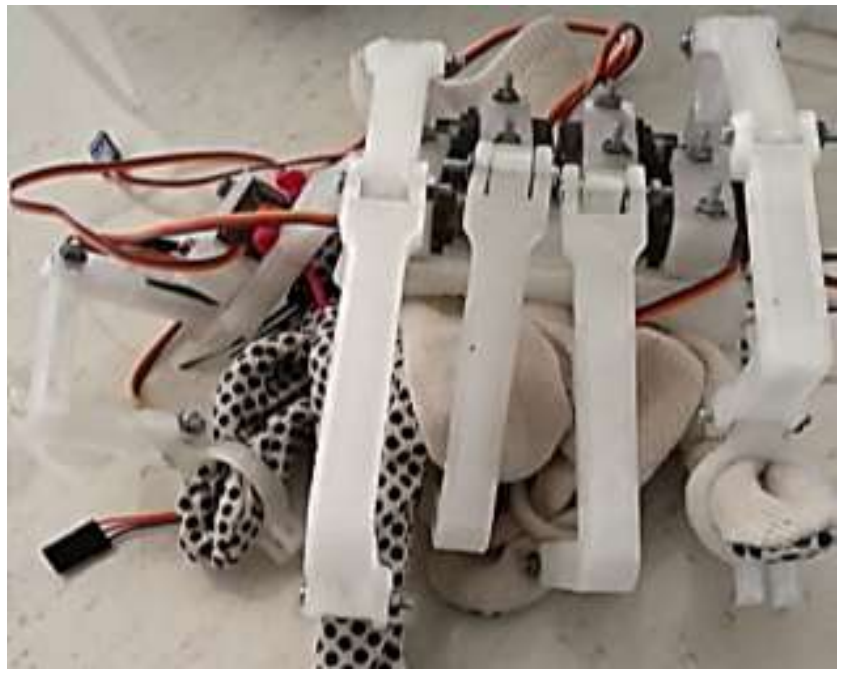


Figura 14. Se muestra el exoesqueleto ensamblado y probado en una mano físicamente, para ver la funcionalidad el mecanismo de cerradura y apertura de los dedos de la mano.

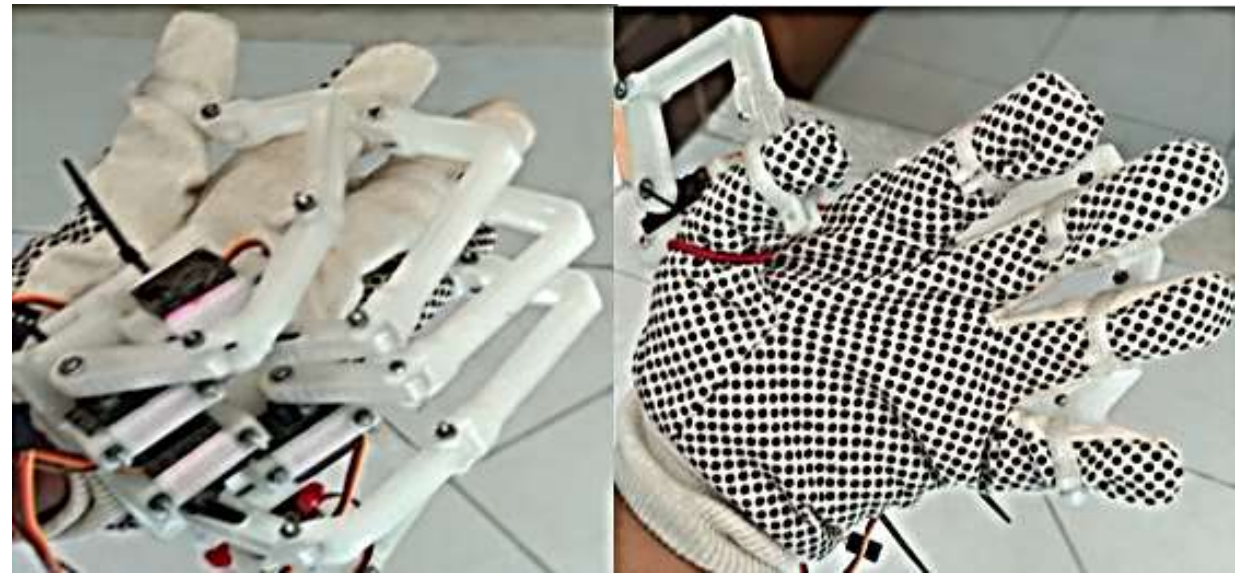

Figura 15. Pruebas y ajustes de la órtesis en físico, considerando ya la segunda etapa del trabajo que sería el sistema motriz y de control.

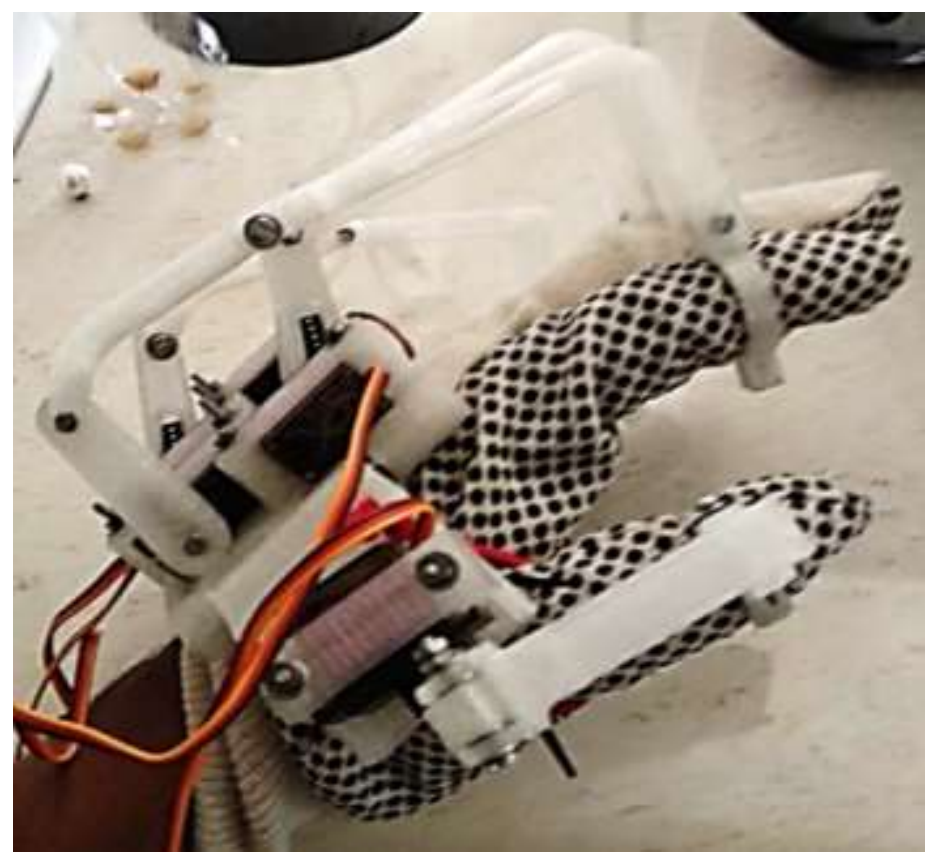

\section{CONCLUSIONES}

El prototipo de órtesis elaborado de material termoplástico (PLA) con circuitos y materiales de fácil acceso, pueden ser adaptadas directamente al paciente y ser fácilmente cambiadas, así como reimpresas cada una de las piezas del prototipo en caso de ruptura o desgastes, el promedio gastos de este proyecto en material llevo un total de $\$ 2100.00$ pesos estando por debajo de promedio de todas las órtesis existentes. 
Este prototipo se puede convertir en una parte integral del proceso de terapia cuando deseamos, la recuperación del movimiento o función en la mano en base al diagnóstico de un especialista.

La órtesis fue diseñada con el fin de ajustarse a cualquier mano que pueda ser recuperable así como su uso correcto y limpieza.

Puede usarse con algunos tipos de guantes del cualquier tipo de tela que permitan la movilidad del esqueleto de la mano. Si la órtesis se moviera mucho dentro los dedos de la mano o molesta se debe ajustar los anillos con sus tornillos y tuercas para aflojar o cerrar el anillo que entre en el dedo de la mano, recomendado el guante para mayor ajuste y comodidad.

Se visualizan de manera virtual los diferentes ángulos del exoesqueleto, para la demostración del mecanismo a través de la simulación, demostrando que tan funcional puede resultar por medio del CAD, ya que el diseño asistido por computadora es lo que más cercano a una simulación de la vida real, para la disminución de errores en una totalidad.

Se aprecia como los extensores de los dedos están extendidos, cuando las palancas que están incorporadas en los servomotores, estos se mueven hacia el punto de juntarse con el soporte, los anillos que van colocados en los dedos, logran crear un tipo de palanca el cual crea la apertura cada uno de las falanges de la mano.

Además, se observa cómo se puede lograr el mismo efecto de empuje, sobre los anillos de los dedos con la retracción de las palancas de los servomotores, cuando estos se encuentran en una posición totalmente inclinadas para el cierre de los dedos de la mano. Lográndose un nivel de funcionamiento de un $99 \%$, siendo como primer prototipo, para llevarlo a la impresión 3D, y ver qué tipo de variables externas afectan este prototipo, lográndolo mejorar con el paso del tiempo.

\section{PROPUESTA PARA TRABAJO FUTURO}

El prototipo de órtesis o férula tiene un segundo proceso que es automatizar su control vía remota a través de una aplicación móvil y a medida que los objetivos terapéuticos cambian, el diseño y/o el uso de este prototipo de órtesis podría evolucionar. Será utilizada durante el período post lesión / post quirúrgico ya que esto dependerá si el paciente tiene mejoría y es estudiado a través de un fisioterapeuta, dando un diagnóstico 
de que tan funcional puede ser el tratamiento de cesiones con el prototipo así como si el paciente es capaz de recuperar la movilidad y el tiempo de recuperación.

En investigaciones más recientes muestran que la combinación entre un tratamiento convencional de movimiento pasivo combinado con uno asistido por un dispositivo robótico genera ganancias significativas no sólo en la mejoría de las condiciones articulares del individuo sino también en su funcionalidad dando claridad que pese a las investigaciones hechas, cada una por separado no obtienen mayores resultados como cuando se combinan, estos dispositivos han de ser seguros, poco complejos y ligeros, para permitir su adecuado uso (Meneses, Peñaloza, Pinzón y Castellanos, 2015).

Se hicieron pruebas de la órtesis en una mano con los tres diferentes tipos de sesiones de ejercicios programados, demostrado que el movimiento de las falanges puede realizarse independiente mente, logrando así la movilidad de estos en más de un 60 por ciento de extensión y flexión de los dedos, probando que es factible para un tratamiento de rehabilitación de mano que lo requiera.

La utilización de este prototipo de órtesis de PLA es de uso corto sin alimentación externa en un máximo 15 minutos, por la duración de las baterías, ya que si existe algún tipo de alimentación secundaria podría utilizarse el tiempo que sea se requiera alimentado a 4.5 volts de corriente directa.

\section{REFERENCIAS BIBLIOGRAFICAS}

“Anet A8” (s.f.). En https://anet3d.mx/products/a8-plus-printer

Arce, C. (2005). Órtesis de miembros superiores clasificación, funciones, prototipos, características e indicaciones. Perú. En https://www.arcesw.com/o_m_s.pdf

Cervantes, J. J. (2019). Manufactura Aditiva (Impresión 3D). México: FI-UNAM:

"Férula Dinámica para mano" (s.f.) En http://ortopedianodal.com/producto/feruladinamica-para-flexion-de-muneca-y-dedos-mod-13b/

"Férula dinámica" (s.f.). En https://www.centralorthomedica.com/brazo.html

Fique, K. A. y Aponte, O. A. (2008). Diseño y construcción de un aditamento ortésico dinámico Para prevención del síndrome de mano caída [Tesis de Maestría]. Bogotá: Universidad de La Salle. http://repository.lasalle.edu.co/bitstream/handle/10185/16669/T44.08\%20F516d. pdf? sequence $=1$ 
Gibson, I., Rosen, D. \& Stucker, B. (2015). Additive Manufacturing Technologies 3D Printing, Rapid Prototyping, and Direct Digital Manufacturing Second Edition. New York: Springer.

Gómez, S. (2010). El Gran Libro de SolidWorks. España: Marcombo.

Hospital del Trabajador ACHS. (2013). Manual de Órtesis. Asociación Chilena de Seguridad: Santiago de Chile. Recuperado de http://www.hospitaldeltrabajador.cl/ht/Comunidad/documentos/Documents/MA NUAL\%20ORTESIS.pdf

Levy, A. E. y Cortés, J. M.. (2003). Ortopodologia y aparato locomotor ortopedia de pie y tobillo. Barcelona: Masson.

Meneses, C., Peñaloza, Y, Pinzón, M. Y. y Castellanos, J. (2015). Aplicación de la terapia robótica para el tratamiento de la mano espástica del adulto con hemiplejía (artículo de revisión). Revista Mexicana de Medicina Física y Rehabilitación 27(3-4) 80-85.

"Mecanismos". (2012). Sites Google. Obtenido de https://sites.google.com/site/gabrielmecanismos/Home/parte-iii/03--transformacion-del-movimiento-giratorio-en-oscilante/3-1---sistema-ruedaexcentrica-biela-palanca

“Órtesis y férulas". (s. f.). En https://cripenalara.com/ortesis-y-ferulas/

Ortiz, J. C.; et al. (16 - 18 de Junio 2016). Férula dinámica para mano con extensor de dedos, pulgar y muñeca [ponencia]. VII Congreso Nacional de Tecnología Aplicada a Ciencias de la Salud. INAOE. Puebla, Puebla. En https://wwwoptica.inaoep.mx/ tecnologia_salud/2016/documentos/memorias/MyT2016_131 _E.pdf

Peña, E.; Ticó, N.; López, J. A.; Abenoza, M. y Romero, G. (s.f.). Exoesqueleto para mano discapacitada con movimiento y sensibilidad, pero sin fuerza. Ortopedia Técnica. España: Universidad Politécnica de Cataluña - Xarxa Assistencial Fundació Althaia

"PLA - blanco" (s.f.). En https://luktec.com/pla-blanco 
Vásquez, Jiménez y otros...

Sánchez-Restrepo, S. (2017). FDM o modelado por deposición fundida, te explicamos todo! En https://www.3dnatives.com/es/modelado-por-deposicionfundida29072015

Zambudio, R. (2009). Protesis, Ortesis Ayudas Externas. España: Elseiver. 